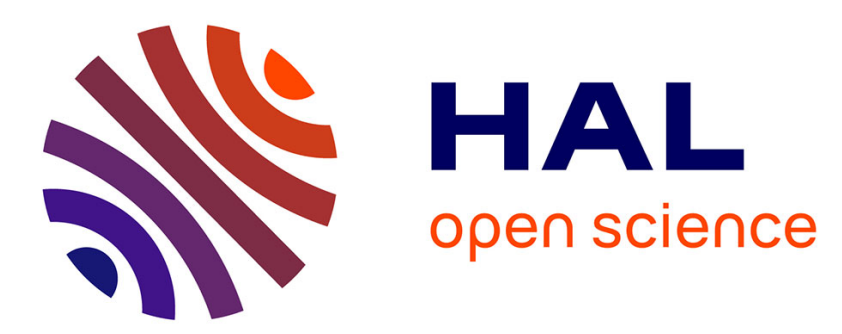

\title{
Optimisation du transfert sur capacité de l'énergie libérée par un élément piézoélectrique en compression \\ H. Ohanessian, P. Gonnard
}

\section{To cite this version:}

H. Ohanessian, P. Gonnard. Optimisation du transfert sur capacité de l'énergie libérée par un élément piézoélectrique en compression. Revue de Physique Appliquée, 1981, 16 (11), pp.647-655. 10.1051/rphysap:019810016011064700 . jpa-00244957

\section{HAL Id: jpa-00244957 https://hal.science/jpa-00244957}

Submitted on 1 Jan 1981

HAL is a multi-disciplinary open access archive for the deposit and dissemination of scientific research documents, whether they are published or not. The documents may come from teaching and research institutions in France or abroad, or from public or private research centers.
L'archive ouverte pluridisciplinaire HAL, est destinée au dépôt et à la diffusion de documents scientifiques de niveau recherche, publiés ou non, émanant des établissements d'enseignement et de recherche français ou étrangers, des laboratoires publics ou privés. 


\title{
Optimisation du transfert sur capacité de l'énergie libérée par un élément piézoélectrique en compression
}

\author{
H. Ohanessian et P. Gonnard \\ Institut National des Sciences Appliquées, Laboratoire de Génie Electrique et Ferroélectricité \\ 20, avenue Albert-Einstein, 69621 Villeurbanne Cedex, France
}

(Reçu le 12 juin 1981, révisé le 29 juillet 1981, accepté le 30 juillet 1981)

\begin{abstract}
Résumé. - Le but de cet article est d'étudier le transfert sur une capacité de l'énergie électrique libérée par une céramique piézoélectrique au cours d'une compression uniaxiale impulsionnelle.

La compression, obtenue par la chute d'une bille d'acier sur l'échantillon, est semblable à celle observée sur les allumeurs piézoélectriques.

Le circuit utilisé est constitué par un pont de diodes de type Graetz débitant sur un condensateur. Ce pont est alimenté par le générateur piézoélectrique soit directement, soit par l'intermédiaire d'une self accordée.

L'étude a été menée en premier lieu sur un modèle simplifié négligeant les variations de compliance de l'élément piézoélectrique, puis généralisée au modèle réel en considérant les équations complètes de la piézoélectricité. Les essais sur des éléments piézoélectriques divers ont confirmé les résultats théoriques.
\end{abstract}

\begin{abstract}
The purpose of the present paper is the study of the transfer on a capacitor of the electrical energy released by a piezoelectric ceramic under a uniaxial stress pulse.

This stress, achieved by the fall on the specimen of a steel ball, is similar to that observed on the piezoelectric impact igniters.

The main circuit is a Graetz-type bridge rectifier loaded by a capacitor. This bridge is either directly feeded by the piezoelectric generator or through a matched self-inductance.

The study was primarily carried out on a simplified model neglecting the variations of compliance, then generalized to the actual model taking into account the piezoelectricity's complete equations. Experiments on various piezoelectric elements confirm the theoretical results.
\end{abstract}

Introduction. - La génération d'énergie électrique à partir de transducteurs piézoélectriques soumis à des impulsions mécaniques uniaxiales constitue une technique classique en plein essor [1,7-9].

Le transfert sur une capacité de l'énergie libérée par l'élément piézoélectrique présente un intérêt double. Il permet d'une part une évaluation rapide et directe des performances du générateur, et d'autre part l'utilisation différée de cette énergie.

Ce travail présente une étude théorique de ce transfert avec la recherche de l'adaptation électrique, complétée par des résultats expérimentaux.

1. Dispositif expérimental (Fig. 1). - Les échantillons à tester se présentent soit sous la forme d'un cylindre de $16 \mathrm{~mm}$ de longueur et $6,35 \mathrm{~mm}$ de diamètre, soit sous celle d'un disque de $1 \mathrm{~mm}$ d'épaisseur et $9 \mathrm{~mm}$ de diamètre. Ils coulissent juste à l'intérieur d'une matrice en afcodur; une graisse aux silicones assure une cohérence parfaite à l'ensemble et supprime le jeu latéral. La transmission et la répartition de l'impulsion de pression sont réalisées par l'intermédiaire d'un marteau cylindrique à tête hémisphérique en acier traité. Divers marteaux sont utilisés suivant les échantillons, afin de produire le signal le plus sinusoïdal. L'ensemble céramique-marteau-matrice est inséré dans une masse cylindrique importante en acier qui élimine les vibrations parasites.

L'impulsion de pression, fournie par la chute d'une bille d'acier d'environ $19 \mathrm{~g}$, est supposée se répartir uniformément sur l'ensemble précédent. Sur les échantillons cylindriques, une hauteur de chute de $30 \mathrm{~cm}$ correspond à une tension à vide d'environ $20 \mathrm{kV}$; c'est la tension que l'on récupère généralement dans les allumeurs piézoélectriques industriels.

2. Développements théoriques. - Nous décomposerons cette étude en deux parties en effectuant d'abord des hypothèses simplificatrices puis en donnant le résultat dans les cas plus complexes. Dans chacun 


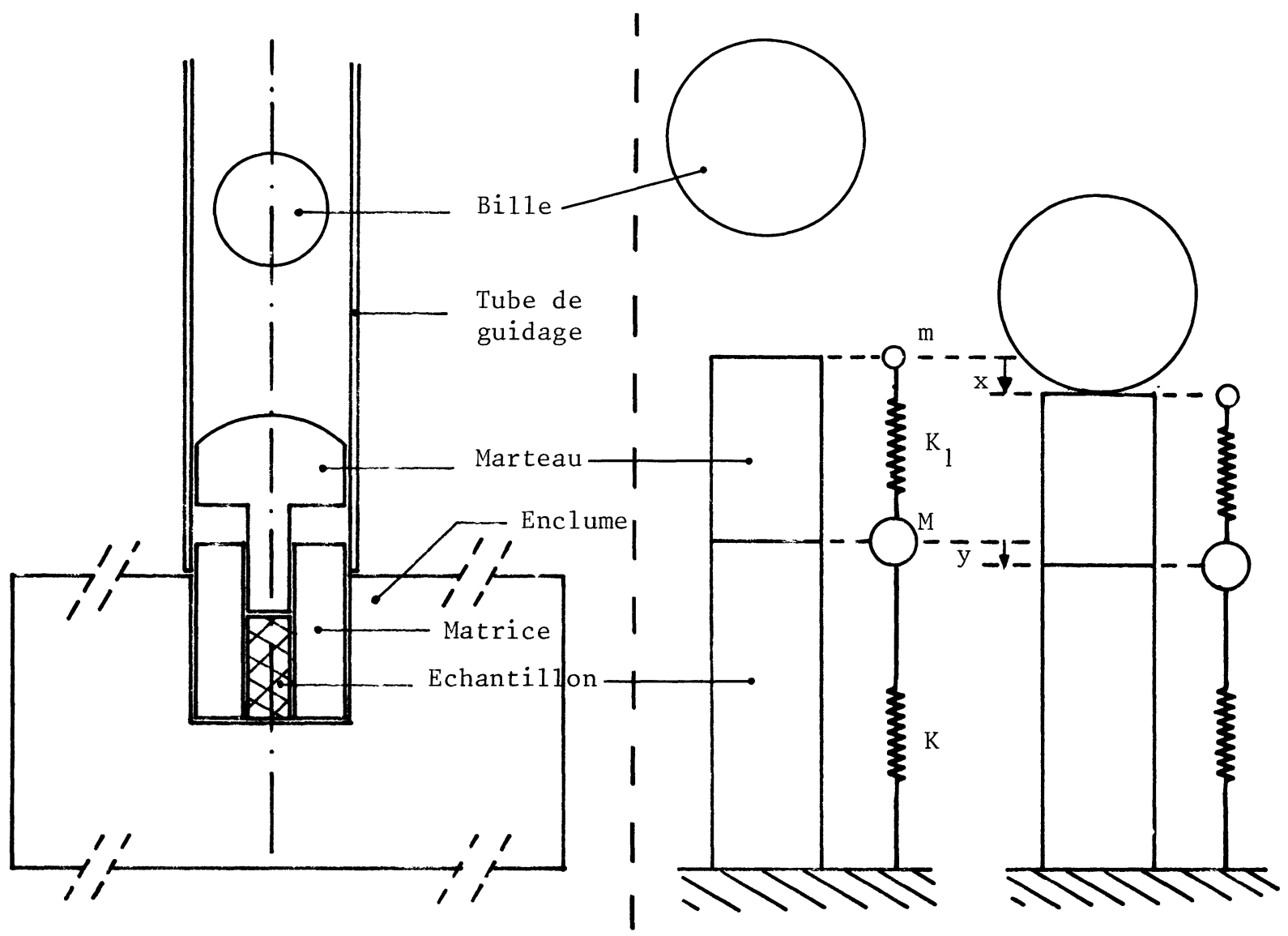

Fig. 1. - Dispositif expérimental.

[Experimental set-up.]

de ces cas, on considère un modèle unidimensionnel dont les propriétés diélectriques et piézoélectriques suivent les lois linéaires classiques [1].

2.1 CéramiQue PiÉZoÉlectrique À COMPLIANCE SUPPOSÉE CONSTANTE PENDANT L'APPLICATION DE LA CONTRAINTE. - On suppose que la compliance reste constante $s^{\mathrm{E}}=s^{\mathrm{D}}=s$ durant l'expérience ce qui correspond à un module d'Young $Y$ constant.

2.1.1 Relations mécaniques. - La bille de masse $m$ est supposée indéformable. Au cours de la contrainte, elle provoque des déformations $x$ sur le marteau de compliance $s_{1}$ et de masse $M$ et des déformations $y$ sur l'échantillon de compliance $s$.

On considère le schéma équivalent simplifié quasi statique de la figure 2 .

Les équilibres dynamiques aux interfaces billemarteau et marteau-échantillon conduisent aux relations suivantes :

$\left\{\begin{array}{l}m \frac{\mathrm{d}^{2} x}{\mathrm{~d} t^{2}}=-K_{1}(x-y) \text { avec } K_{1}=\frac{A_{1}}{s_{1} l_{1}} \\ F=M \frac{\mathrm{d}^{2} y}{\mathrm{~d} t^{2}}-K_{1}(x-y)=-K y \text { avec } K=\frac{A}{s l}\end{array}\right.$
Fig. 2. - Compression et équivalent mécanique.

[Compressive stress and mechanical equivalent circuit.]

$A$ et $l$ représentent la surface et l'épaisseur de l'échantillon, $A_{1}$ et $l_{1}$ celles du marteau assimilé à un cylindre.

$\mathrm{La}$ résolution de ce système d'équations différentielles du $2^{\mathrm{e}}$ ordre conduit à une impulsion de pression $T$ de la forme :

$$
T=\frac{F}{A}=\frac{y}{s l}=\frac{y_{0}}{s l}\left(\sin \omega^{\prime} t-\frac{\omega^{\prime}}{\omega^{\prime \prime}} \sin \omega^{\prime \prime} t\right) .
$$

Parmi les configurations particulières de ce système, on peut envisager les cas limites suivants :

- $K \gg K_{1}$. Ceci correspond à un marteau beaucoup plus déformable que l'échantillon; cette configuration conduit à une forme d'impulsion sinusoïdale sur le marteau

$$
x=\frac{v_{0}}{\omega_{1}} \sin \omega_{1} t
$$

l'échantillon ne se déforme pratiquement pas.

$$
y \# 0 \text {. }
$$

$v_{0}$ est la vitesse de la bille à l'interface bille-enclume.

- $K \ll K_{1}$. C'est le cas d'un marteau indéformable. La pulsation $\omega^{\prime}$ devient alors faible et l'impulsion de pression ainsi que la déformation du marteau ont une forme qui se rapproche d'une sinusoïde. 
Toutefois, pour l'impulsion sur l'échantillon, la pente de la courbe est nulle à l'origine, ce qui provoque un trainage de l'onde autour de ce point. C'est un cas simple qui a déjà été étudié [2].

En jouant sur ces paramètres, il est possible de produire sur l'échantillon une impulsion de pression propre, peu affectée par les termes en $\sin \omega^{\prime \prime} t$.

On peut donc, en première approximation, donner l'équation sous la forme

$$
T=T_{\mathrm{M}} \sin \omega t
$$

pour des temps variant entre 0 et $t_{0}=\pi / \omega, t_{0}$ représentant la durée totale de l'impulsion de pression.

2.1.2 Equations piézoélectriques. - 1) Dans le montage d'étude de la figure 3 (interrupteur I fermé) on considère un générateur piézoélectrique cylindrique de constante diélectrique $\varepsilon^{T}$ et de capacité

$$
C=\varepsilon^{T} \frac{A}{l} .
$$

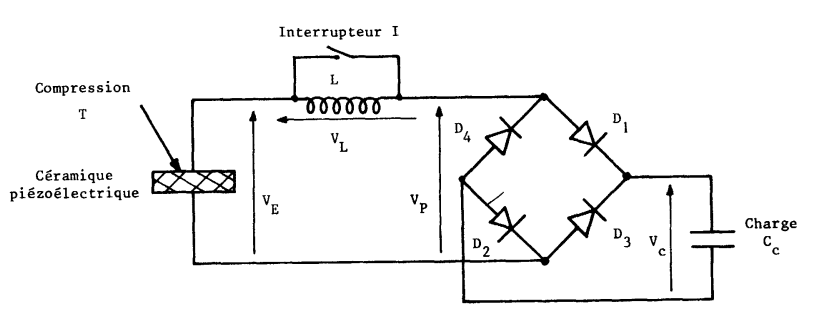

Fig. 3. - Dispositif de mesure.

[Measuring device.]

Pour simplifier les relations, on définit pour le condensateur de charge $C_{\mathrm{c}}$, une constante diélectrique $\varepsilon_{\mathrm{c}}$ telle que

$$
C_{\mathrm{c}}=\varepsilon_{\mathrm{c}} \frac{A}{l} .
$$

On supposera que les diodes haute tension ont des caractéristiques parfaites (en particulier temps de recouvrement nul). Etudions alors l'évolution de la charge du condensateur.

2) Diodes conductrices. La céramique joue le rôle de générateur et fournit une densité de charges dont la variation s'exprime par la relation suivante [3, 4] :

$$
\mathrm{d} D=\varepsilon^{T} \mathrm{~d} E+d_{33} \mathrm{~d} T
$$

$d_{33}$ est le coefficient piézoélectrique et $E$ le champ électrique aux bornes de la céramique.

Sil'un des couples $\left(D_{1}, D_{2}\right)$ ou $\left(D_{3}, D_{4}\right)$ est en conduction, cette variation de charges est transmise au condensateur $C_{\mathrm{c}}$ qui se comporte en récepteur, ce qui entraîne :

$$
\mathrm{d} D=-\varepsilon_{\mathrm{c}} \mathrm{d} E .
$$

Il vient alors :

$$
\mathrm{d} E=-\frac{d_{33}}{\varepsilon^{T}+\varepsilon_{\mathrm{c}}} \mathrm{d} T
$$

d'où l'on tire l'expression du champ électrique :

$$
E=-\frac{d_{33}}{\varepsilon^{T}+\varepsilon_{\mathrm{c}}} T+E_{0} .
$$

3) Diodes bloquées. La céramique est en circuit ouvert, d'où $\mathrm{d} D=0$ ce qui entraîne la variation de champ

$$
\mathrm{d} E=-\frac{d_{33}}{\varepsilon^{T}} \mathrm{~d} T
$$

et un champ

$$
E=-\frac{d_{33}}{\varepsilon^{T}} T+B
$$

2.1.3 Adaptation électrique. - 1) Envisageons d'abord le cas où la contrainte est supposée sinusoïdale pure sur une demi-période puis s'annule.

Pendant la montée de la compression, jusqu'au temps $t_{\mathrm{M}}$, les diodes $D_{1}$ et $D_{2}$ conduisent ; d'après (3) le champ est de la forme :

$$
E_{1}=-\frac{d_{33}}{\varepsilon^{T}+\varepsilon_{\mathrm{c}}} T_{\mathrm{M}} \sin \omega t \text { avec } E_{1}(0)=0 .
$$

Au temps $t_{M}=\pi / 2 \omega$, le champ aux bornes de la céramique atteint la valeur

$$
E_{\mathrm{M}}=-\frac{d_{33}}{\varepsilon^{T}+\varepsilon_{\mathrm{c}}} T_{\mathrm{M}} .
$$

La compression diminuant, les diodes se bloquent et la relation (4) s'applique avec la condition aux limites :

$$
t=t_{\mathrm{M}}, \quad E=E_{\mathrm{M}}
$$

il vient :

$$
E_{2}=-\frac{d_{33}}{\varepsilon^{T}}\left[\sin \omega t-\frac{\varepsilon_{\mathrm{c}}}{\varepsilon^{T}+\varepsilon_{\mathrm{c}}}\right] T_{\mathrm{M}} .
$$

Le champ aux bornes de la céramique décroît, devient négatif et atteint la valeur $-E_{\mathrm{M}}$ au temps

$$
t_{1}=\frac{1}{\omega} \arcsin \frac{\varepsilon_{\mathrm{c}}-\varepsilon^{T}}{\varepsilon_{\mathrm{c}}+\varepsilon^{T}} .
$$

Les diodes $D_{3}$ et $D_{4}$ deviennent alors conductrices et le champ reprend la forme de la relation (3); en utilisant la condition aux limites pour $t=t_{1}$

$$
E_{3}=-\frac{d_{33}}{\varepsilon^{T}+\varepsilon_{\mathrm{c}}} T_{\mathrm{M}}\left[\sin \omega t-\frac{2 \varepsilon_{\mathrm{c}}}{\varepsilon^{T}+\varepsilon_{\mathrm{c}}}\right]
$$


il atteint sa valeur maximale $E_{3 \mathrm{M}}$ lorsque la compression s'annule; la charge du condensateur s'écrit alors :

$$
V_{\mathrm{MM}}=l \frac{d_{33} T_{\mathrm{M}}}{\varepsilon^{T}+\varepsilon_{\mathrm{c}}} \cdot 2 \frac{\varepsilon_{\mathrm{c}}}{\varepsilon^{T}+\varepsilon_{\mathrm{c}}}=-l E_{3 \mathrm{M}} .
$$

La figure 4 schématise l'évolution théorique de la contrainte $T$, du champ $E$ aux bornes de la céramique et de la densité de courant $j$ débitée par la céramique.

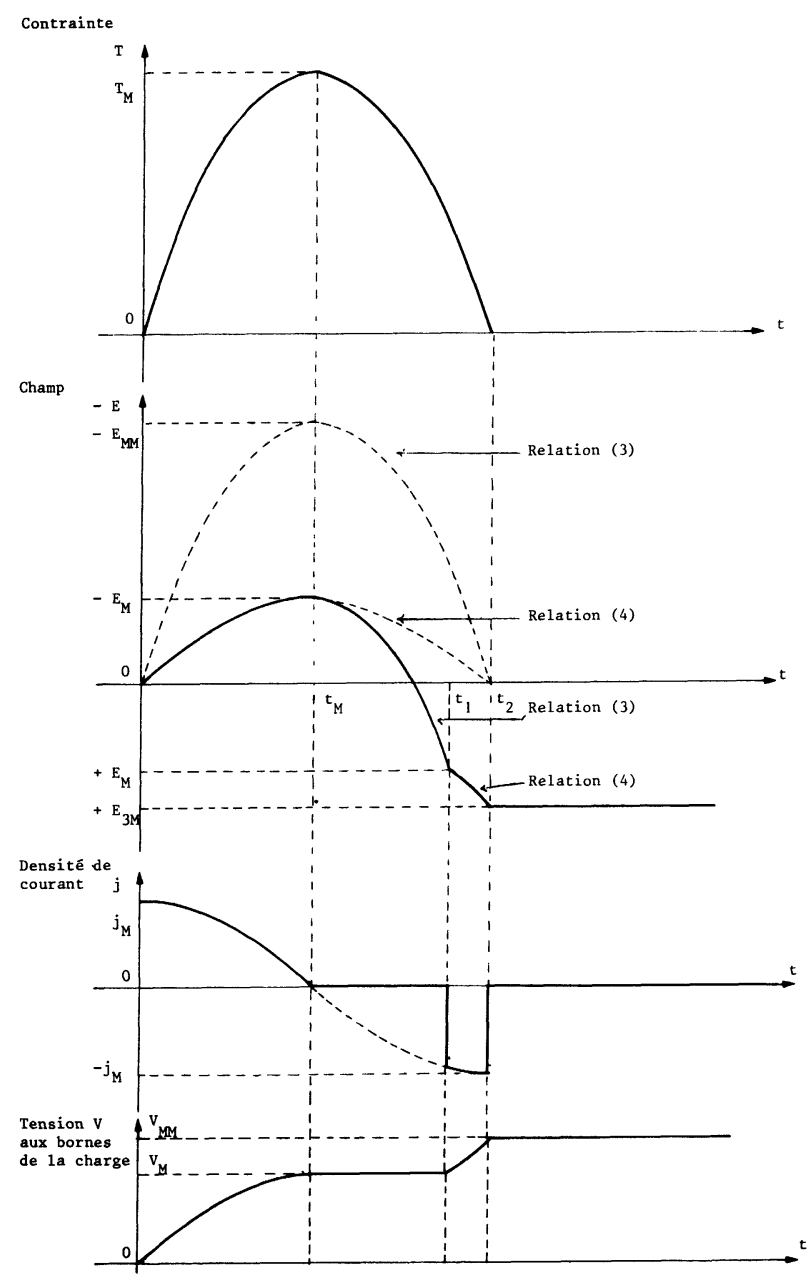

Fig. 4. - Chronogrammes théoriques du montage sans self.

[Theoretical time-dependent plots for the circuit without the inductance-coil.]

L'énergie libérée par la céramique sous la compression se retrouve, aux pertes près, dans la capacité :

$$
W_{\max }=\frac{1}{2} C_{\mathrm{c}} V_{\mathrm{MM}}^{2}
$$

et la densité d'énergie s'écrit :

$$
W=\frac{W_{\max }}{v^{\prime}}=2 F T_{\mathrm{M}}^{2} \frac{\alpha^{3}}{(1+\alpha)^{4}}, \quad v^{\prime}=A 1
$$

expression dans laquelle le facteur de mérite $F$ s'exprime par

$$
F=\frac{d_{33}^{2}}{\varepsilon^{T}}
$$

et $\alpha$ représente le rapport des capacités

$$
\alpha=\frac{C_{\mathrm{c}}}{C}=\frac{\varepsilon_{\mathrm{c}}}{\varepsilon^{T}} .
$$

Le maximum de l'énergie vaut

$$
\frac{W_{\mathrm{MM}}}{v^{\prime}}=0,422 \cdot \frac{1}{2} F T_{\mathrm{M}}^{2}=0,422 \frac{W_{0}}{v^{\prime}}
$$

et s'obtient pour $\alpha=3$ soit

$$
C_{\mathrm{c}}=3 C
$$

On trouve un facteur 0,422 par rapport à l'énergie $W_{0} / v^{\prime}$ libérable par la céramique après application d'une contrainte statique égale à $T_{\mathrm{M}}$.

Remarque : Si l'on considère une compression de la forme (2), les expressions des champs dans les relations (3) et (4) sont inchangées; on retrouve alors les conditions d'adaptation (5).

2.1.4 Améliorations apportées par une inductance série. - On utilise le montage électrique représenté sur la figure 3 (interrupteur ouvert). On prend la notation réduite $\lambda=L \frac{A}{l}$. Tant que $D_{1}$ et $D_{2}$ conduisent, un courant $j=\mathrm{d} D / \mathrm{d} t$ charge le condensateur $C_{\mathrm{c}}$ et l'équation différentielle de la charge prend la forme :

$$
\lambda \frac{\varepsilon^{T} \varepsilon_{\mathrm{c}}}{\varepsilon^{T}+\varepsilon_{\mathrm{c}}} \frac{\mathrm{d}^{2} j}{\mathrm{~d} t^{2}}+j=\frac{d_{33} \varepsilon_{\mathrm{c}}}{\varepsilon^{T}+\varepsilon_{\mathrm{c}}} \frac{\mathrm{d} T}{\mathrm{~d} t} .
$$

En supposant une compression de forme sinusoïdale $T=T_{\mathrm{M}} \sin \omega t$, on obtient la solution générale suivante avec la condition aux limites $: t=0, j=0$

$$
j=\frac{\varepsilon_{\mathrm{eq}}}{\varepsilon^{T}} \frac{d_{33} \omega T_{\mathrm{M}}}{1-\frac{\omega^{2}}{\omega^{\prime 2}}}\left[\cos \omega t-\cos \omega^{\prime} t\right]
$$

avec

$$
\omega^{\prime 2}=\frac{1}{\lambda \varepsilon_{\mathrm{eq}}}=\frac{1}{L C_{\mathrm{eq}}} \quad \text { et } \quad \varepsilon_{\mathrm{eq}}=\frac{\varepsilon^{T} \varepsilon_{\mathrm{c}}}{\varepsilon^{T}+\varepsilon_{\mathrm{c}}} .
$$

On se place dans le cas particulier de la résonance où $\omega \simeq \omega^{\prime}$ et

$$
j=\frac{\varepsilon_{\mathrm{eq}}}{\varepsilon^{T}} \frac{\omega^{\prime 2}}{2} d_{33} T_{\mathrm{M}} t \sin \omega^{\prime} t .
$$

A l'instant $t_{1}=\pi / \omega^{\prime}: j=0, V_{\mathrm{p}}=V_{\mathrm{c} 1}$ et les diodes se bloquent; la self s'oppose à cette diminution brutale 
du courant et crée une différence de potentiel $V_{\mathrm{L}}=\lambda l \mathrm{~d} j / \mathrm{d} t$ qui se répercute aux bornes du pont. Lorsque cette tension atteint la valeur $-V_{\mathrm{c} 1}$ (pour $\left.t_{2} \simeq t_{1}\right), D_{3}$ et $D_{4}$ conduisent. Mais la compression est alors achevée d'où :

$$
\lambda \varepsilon^{T} \frac{\mathrm{d}^{2} j}{\mathrm{~d} t^{2}}+\frac{\varepsilon_{\mathrm{c}}+\varepsilon^{T}}{\varepsilon_{\mathrm{c}}} j=0
$$

qui conduit à la valeur du courant :

$$
j=\frac{V_{\mathrm{L} 1}+2 V_{\mathrm{c} 1}}{\omega^{\prime} \lambda l} \sin \omega^{\prime}\left(t-t_{2}\right) .
$$

Le condensateur se charge à nouveau jusqu'au maximum en $t=t_{3}$

$$
V_{\mathrm{c} 3}=\frac{\pi}{2} l \frac{d_{33} T_{\mathrm{M}}}{\left(\varepsilon^{T}+\varepsilon_{\mathrm{c}}\right)^{2}}\left(3 \varepsilon_{\mathrm{c}}-\varepsilon^{T}\right) .
$$

L'énergie récupérée dans la charge au temps $t_{3}$ s'écrit :

$$
\frac{W_{\mathrm{c} 3}}{v^{\prime}}=\frac{1}{2} C_{\mathrm{c}} V_{\mathrm{c} 3}^{2}=\frac{\pi^{2}}{8} d_{33}^{2} T_{\mathrm{M}}^{2} \frac{\varepsilon_{\mathrm{c}}\left(3 \varepsilon_{\mathrm{c}}-\varepsilon^{T}\right)^{2}}{\left(\varepsilon_{\mathrm{c}}+\varepsilon^{T}\right)^{4}}
$$

Cette fonction passe par un maximum pour :

$C_{\mathrm{c}}=3,91 \quad C \frac{W_{\mathrm{cM}}}{v^{\prime}}=1,91\left(\frac{1}{2} F T_{\mathrm{M}}^{2}\right)=1,91 \frac{W_{0}}{v^{\prime}}$.

A contrainte maximum $T_{\mathrm{M}}$ identique, cette valeur représente un gain de 4,53 par rapport à l'énergie d'adaptation obtenue dans un schéma sans self.

Les chronogrammes des diverses tensions présentées figure 5 pour un échantillon type 801 confirment l'étude théorique précédente.

2.2 MODÈLE RÉEL D'UNE CÉRAMIQUe DONT LA COMPLIANCE EST VARIABLE. - Le champ électrique et l'induction électrique aux bornes de la céramique variant au cours de la compression, la compliance $s$ de celle-ci n'est pas constante. Il faudra donc introduire une relation représentant la dépendance du champ électrique avec la compliance réelle du matériau.

2.2.1 Etablissement des équations. - On utilise maintenant les deux équations piézoélectriques [3]

$$
\begin{aligned}
& \mathrm{d} D=\varepsilon^{T} \mathrm{~d} E+d_{33} \mathrm{~d} T=-\varepsilon_{\mathrm{c}} \mathrm{d} E \\
& \mathrm{~d} T=\mathrm{c}^{E} \mathrm{~d} S-\mathrm{e}^{T} \mathrm{~d} E
\end{aligned}
$$

où

$$
\mathrm{d} S=\frac{\mathrm{d} y}{l}
$$

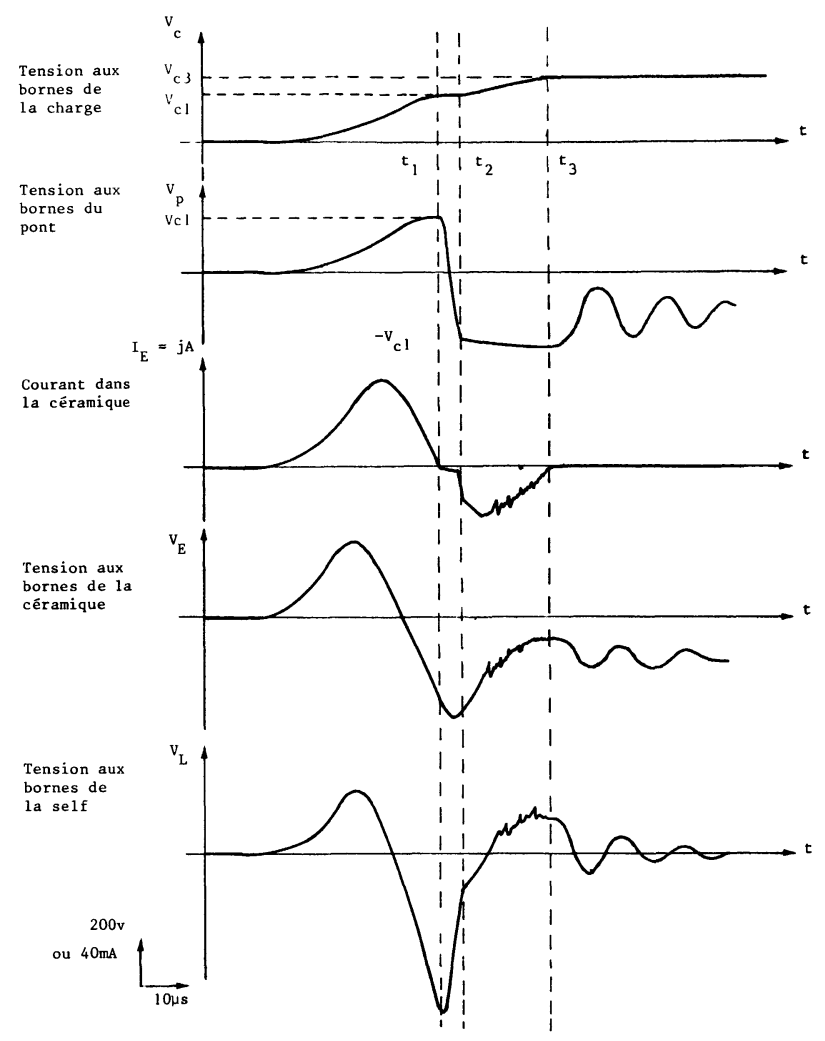

Fig. 5. - Evolution avec le temps : Céramique $n^{\circ} 801$.

[Waveforms for the ceramic $\mathbf{n}^{\circ} 801$.]

représente la variation de déformation relative (Fig. 2),

$$
c^{E}=\frac{1}{s^{E}}
$$

le module d'Young à champ constant du matériau,

$$
e^{T}=\frac{\varepsilon^{T}-\varepsilon^{S}}{d_{33}}
$$

le coefficient de contrainte à déformation nulle.

De ces équations on tire l'équation différentielle

$$
\frac{l M}{A c^{E}}\left(\varepsilon^{S}+\varepsilon_{\mathrm{c}}\right) \frac{\mathrm{d}^{3} E}{\mathrm{~d} t^{3}}+\left(\varepsilon_{\mathrm{c}}+\varepsilon^{T}\right) \frac{\mathrm{d} E}{\mathrm{~d} t}=0 .
$$

Les conditions limites sont : $t=0, E=0$ et $v=v_{1}$, $v_{1}$ vitesse initiale du marteau.

On en déduit la variation du champ électrique lors de la charge du condensateur :

$$
E=-\frac{d_{33} v_{1} \sin \omega_{0} t}{\sqrt{\frac{l A}{M c^{E}}\left(\varepsilon^{T}+\varepsilon_{c}\right)\left(\varepsilon^{S}+\varepsilon_{c}\right)}}
$$

avec

$$
\omega_{0}^{2}=\frac{A c^{E}}{l M} \cdot \frac{\varepsilon^{T}+\varepsilon_{\mathrm{c}}}{\varepsilon^{S}+\varepsilon_{\mathrm{c}}}
$$


2.2.2 Conditions d'adaptation. - En définissant la contrainte maximum atteinte en court-circuit par

$$
T_{0}^{E}=v_{1} \sqrt{\frac{M c^{E}}{A l}}
$$

on réitère l'étude précédente qui conduit à un champ électrique maximum :

$$
E_{3 \mathrm{M}}=\frac{2 d_{33} T_{0}^{E}}{\sqrt{\left(\varepsilon^{T}+\varepsilon_{\mathrm{c}}\right)\left(\varepsilon^{S}+\varepsilon_{\mathrm{c}}\right)}} \times
$$

$$
\left[1-\frac{\varepsilon^{T}}{\sqrt{\left(\varepsilon^{T}+\varepsilon_{c}\right)\left(\varepsilon^{S}+\varepsilon_{c}\right)}}\right] .
$$

On pose $\alpha=\varepsilon_{\mathrm{c}} / \varepsilon^{T}$ et le coefficient de couplage $k_{33}$ de la céramique est défini par :

$$
k_{33}^{2}=k^{2}=1-\frac{\varepsilon^{S}}{\varepsilon^{T}}=\frac{d_{33}^{2}}{\varepsilon^{T} s^{E}} .
$$

Le maximum d'énergie récupérée s'écrit alors :

$$
\begin{aligned}
& \frac{W_{\max }}{v^{\prime}}=2 F\left(T_{0}^{E}\right)^{2} \frac{\alpha}{(1+\alpha)\left(1-k^{2}+\alpha\right)} \times \\
& {\left[1-\frac{1}{\sqrt{(1+\alpha)\left(1-k^{2}+\alpha\right)}}\right]^{2} . }
\end{aligned}
$$

Cette expression devient, en remplaçant $F$ et $T_{0}^{E}$ par leurs valeurs :

$$
\begin{aligned}
\frac{W_{\max }}{v^{\prime}}=\frac{1}{2} \frac{M v_{1}^{2}}{v^{\prime}} \frac{4 k^{2} \alpha}{(1+\alpha)\left(1-k^{2}+\alpha\right)} \times \\
\quad\left[1-\frac{1}{\sqrt{(1+\alpha)\left(1-k^{2}+\alpha\right)}}\right]^{2}
\end{aligned}
$$

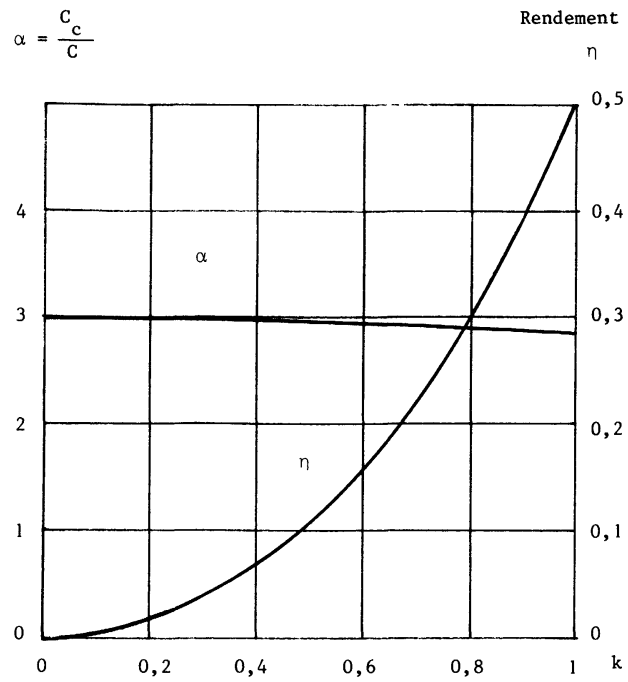

Fig. 6. - Caractéristiques d'adaptation capacitive.

[Matching characteristics as a function of the coupling factor.] $\frac{1}{2} M v_{1}^{2}$ étant l'énergie cinétique incidente sur la céramique, le terme restant représente le rendement $\eta$ de la conversion mécanoélectrique. Les courbes de la figure 6 donnent la valeur de la capacité d'adaptation lorsque $k$ varie de 0 à 1 et celle du rapport $\eta$ :

$$
\eta=\frac{W_{\max }}{v^{\prime}} / \frac{1}{2} \frac{M v_{1}^{2}}{v^{\prime}} .
$$

On remarque que la capacité d'adaptation varie très peu (de $3 C$ à 2,885 $C$ ) alors que l'énergie maximum passe de 0 pour $k=0$ à $0,506 \cdot \frac{1}{2} M v_{1}^{2}$ pour $k=1$.

2.2.3 Cas général d'une enclume réelle non parfaite. - En plus de la compliance réelle du matériau, il convient de tenir compte de la compressibilité de l'enclume comme dans le paragraphe 2.1 et la figure 2 .

Aux deux équations piézoélectriques (8), on associera maintenant les deux équations mécaniques (1).

$$
\begin{gathered}
\varepsilon^{T} \mathrm{~d} E+d_{33} \frac{\mathrm{d} F}{A}=-\varepsilon_{\mathrm{c}} \mathrm{d} E \\
C^{E} \frac{\mathrm{d} y}{l}-\frac{\varepsilon^{T}-\varepsilon^{S}}{d_{33}} \mathrm{~d} E=\frac{\mathrm{d} F}{A} \\
m \frac{\mathrm{d}^{2} x}{\mathrm{~d} t^{2}}=-\frac{A_{1}}{s_{1} l_{1}}(x-y) \\
M \frac{\mathrm{d}^{2} y}{\mathrm{~d} t^{2}}-\frac{A_{1}}{s_{1} l_{1}}(x-y)=F .
\end{gathered}
$$

La résolution de ce système s'effectue en utilisant des méthodes numériques. Elle fait apparaître de légères modifications sur les conditions d'adaptation et les valeurs maximales d'énergie.

3. Résultats expérimentaux et discussion. - Nous avons vérifié les formules théoriques par des essais sur plusieurs types d'échantillons.

3. 1 ETUDES DES CÉRAMIQUES FINES. - Les montages expérimentaux présentent des capacités parasites d'une dizaine de picofarads; nous avons déterminé avec précision ces capacités pour chaque type de montage. Toutefois, pour que l'influence des variations de ces capacités soit faible, nous avons vérifié les formules sur des céramiques fines dont la capacité propre est élevée.

Nous avons testé une céramique de laboratoire (type 801) [5] d'épaisseur $e=1 \mathrm{~mm}$, de diamètre $\varnothing=9,35 \mathrm{~mm}$ et de capacité $C=1180 \mathrm{pF}$.

3.1.1 Pour le montage sans self de la figure 3, nous avons relevé les courbes $V_{\mathrm{c}}, V_{\mathrm{E}}$ et $I_{\mathrm{c}}=f(t)$ (Fig. 7) au moyen d'une sonde de tension Tektronix type 6015 et d'une sonde de courant Tektronix P6042. Elles sont en bonne concordance avec les courbes théoriques. La courbe $V_{\mathrm{c}}=f\left(C_{\mathrm{c}}\right)$ permet de tracer la variation $W=f\left(C_{\mathrm{c}}\right)$ (Fig. 8) et de déterminer l'énergie d'adaptation $W_{\mathrm{MM}}=540 \mu \mathrm{J}$. La capacité pratique d'adaptation $C_{\mathrm{c}}=4000 \mathrm{pF}$ n'est pas déter- 


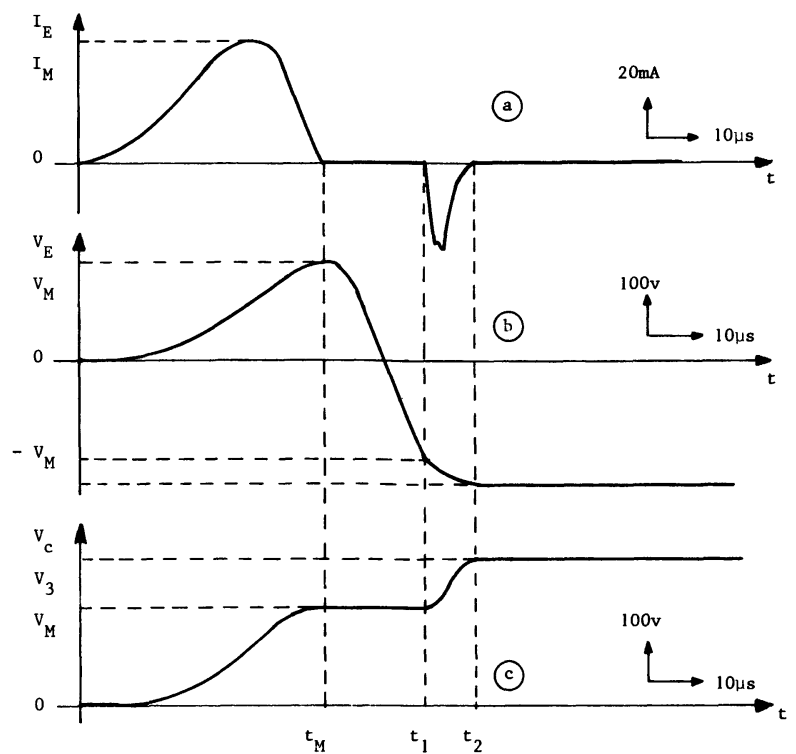

Fig. 7. - Evolution avec le temps. Céramique no $801: a) \mathrm{du}$ courant $I_{\mathrm{E}}$ dans la céramique ; $b$ ) de la tension $V_{\mathrm{E}}$ aux bornes de la céramique ; $c$ ) de la tension $V_{\mathrm{c}}$ aux bornes de la charge $C_{\mathrm{c}}=4400 \mathrm{pF}$.

[Variations against time for the ceramic $\mathrm{n}^{\circ} 801: a$ ) current $I_{\mathrm{E}}$ in the ceramic; $b$ ) voltage $V_{\mathrm{E}}$ across the ceramic; $c$ ) voltage $V_{\mathrm{c}}$ across the load $C_{\mathrm{c}}=4400 \mathrm{pF}$.]

v

kvolts

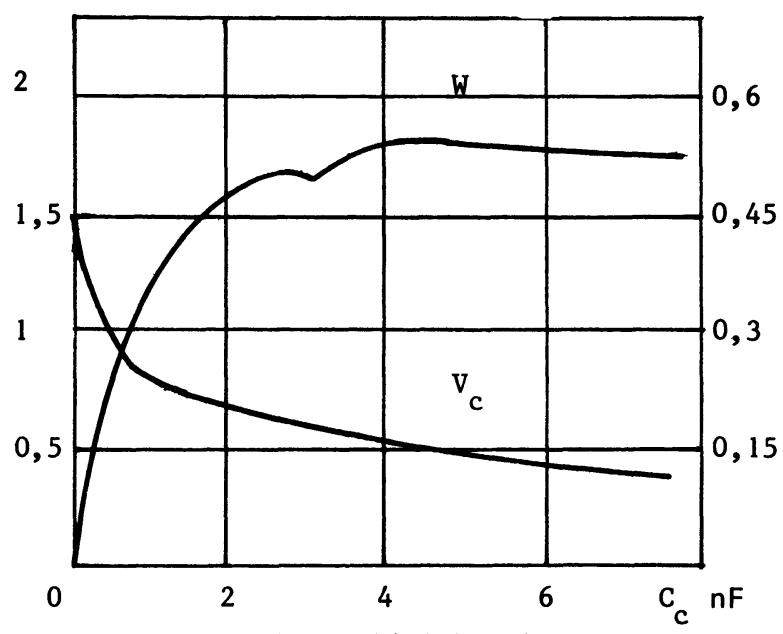

Fig. 8. - Recherche de la capacité d'adaptation.

[Determination of the matching capacitor.]

minée avec une grande précision car la courbe $V_{\mathrm{c}}\left(C_{\mathrm{c}}\right)$ présente un maximum très plat pour une grande excursion de la capacité de charge. Ceci peut expliquer le décalage avec la valeur théorique qui serait de $3540 \mathrm{pF}$. Ces courbes ont été relevées pour une hauteur de chute de $14 \mathrm{~cm}$ afin de ne pas provoquer le claquage de la céramique.

3.1.2 La courbe de la tension à vide $V_{0}=f(t)$ (Fig. 9) en absence de self et de pont redresseur permet de déterminer la durée de la compression et donc la pulsation fondamentale $\omega$ de celle-ci. Dans notre cas la valeur approximative de la demi-période de $40 \mu$ s conduit à une self d'adaptation de l'ordre de $100 \mathrm{mH}$.
V

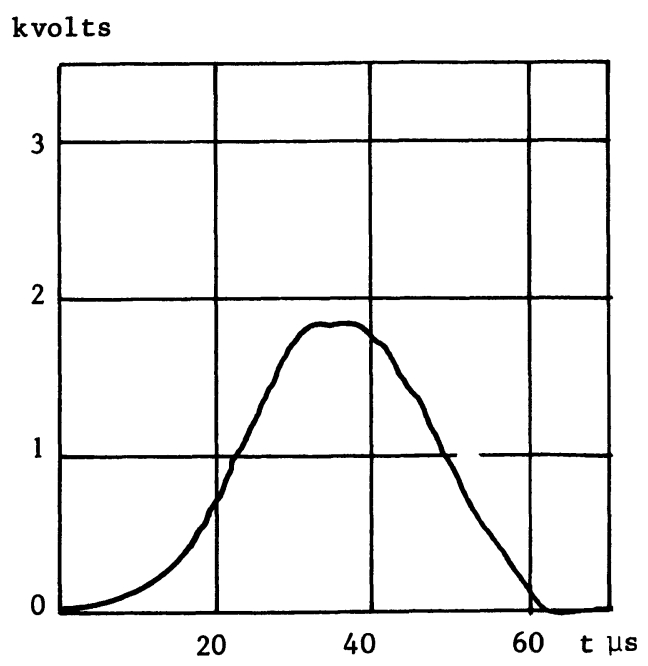

Fig. 9. - Tension à vide.

[Open-circuit voltage.]

Nous vérifions cette valeur à partir du tracé de l'énergie maximale en fonction de $L$ pour la capacité d'adaptation. La courbe correspondante confirme une self d'accord de $L=75 \mathrm{mH}$ (Fig. 10). Pour cette valeur de la self d'accord, les tracés de l'évolution de

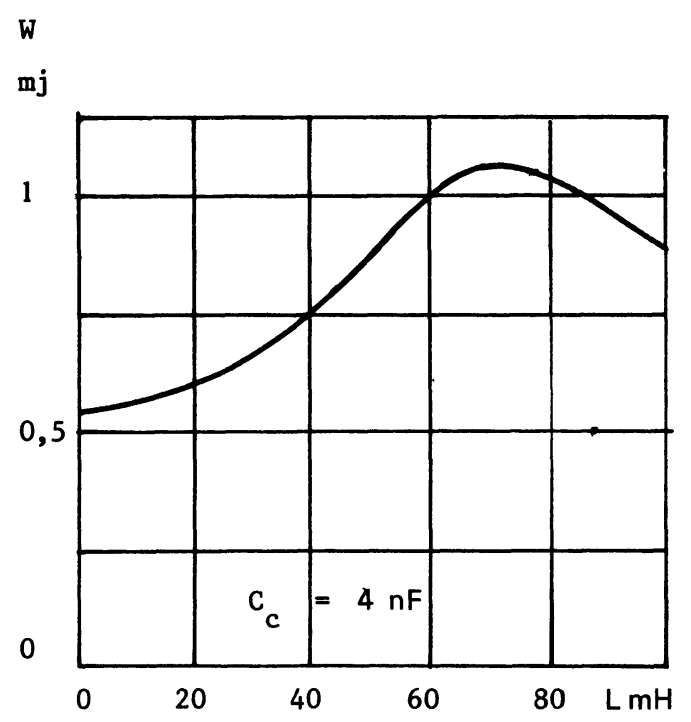

Fig. 10. - Recherche de la self d'accord.

[Determination of the tuning inductance.]

la tension maximum et de l'énergie récupérée avec la capacité de charge (Fig. 11) suivent parfaitement les courbes théoriques précédentes. Ils montrent en particulier un changement de pente pour une capacité $C_{\mathrm{c}} \simeq C$ correspondant à celle de la céramique. On note également l'adaptation pour $C_{\mathrm{c}}=4800 \mathrm{pF}$ alors que la valeur théorique serait de $4613 \mathrm{pF}$.

L'énergie recueillie à ce double accord (capacité et self) est de $1280 \mu \mathrm{J}$ soit 2,4 fois supérieure à celle recueillie en plaçant seulement une capacité adaptée. Si les compressions appliquées à l'échantillon étaient 


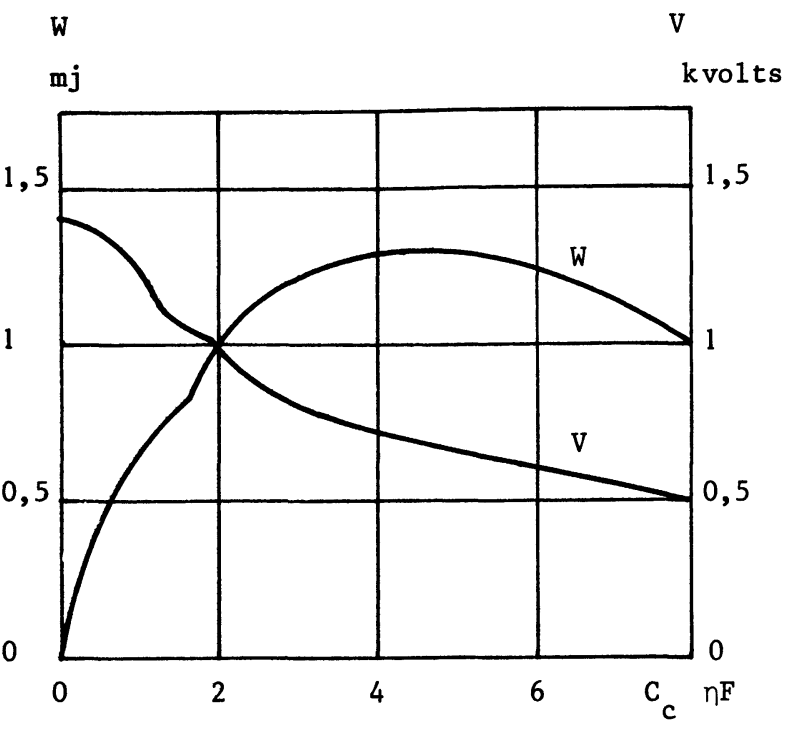

Fig. 11. - Caractéristiques d'adaptation.

[Matching characteristics.]

identiques dans les deux cas, les relations (4) et (7) montrent que le gain d'énergie serait dans un rapport $1,91 / 0,422=4,5$.
3.2 ESSAIS SUR CÉRAMIQUES ÉPAISSES. - La capacité des céramiques industrielles de dimensions standard $e=16 \mathrm{~mm}, \varnothing=6,35 \mathrm{~mm}$ étant plus faible (une trentaine de $\mathrm{pF}$ généralement), la correction de capacité parasite sera beaucoup plus importante que pour les céramiques fines; dans notre cas la capacité parasite mesurée est de $12 \mathrm{pF}$.

Nous avons également constaté des variations de performances de certains échantillons après de multiples compressions : aussi avons-nous choisi un critère de stabilité de nos céramiques; il est défini par la stabilité du coefficient piézoélectrique $d_{33}$ après un test de 500 chocs consécutifs à une fréquence de répétition de 10 chocs à la minute.

Les résultats des essais comparatifs sur céramiques industrielles de type PXE 21, PXE 41, KANEMATSU et sur céramiques fabriquées au laboratoire de type 738, 753 et 1360 (notées ${ }^{*}$ ) sont donnés tableau I. On note que le rapport $C_{\mathrm{c}}^{\prime} / C\left(C_{\mathrm{c}}^{\prime}=C_{\mathrm{c}}+C_{\text {parasite }}\right)$ reste proche de la valeur théorique calculée.

Une comparaison avec le facteur de couplage $k$ mesuré pour chaque céramique montre que l'énergie maximum varie généralement bien comme ce facteur. Or les relations piézoélectriques [6] indiquent que le

Tableau I.

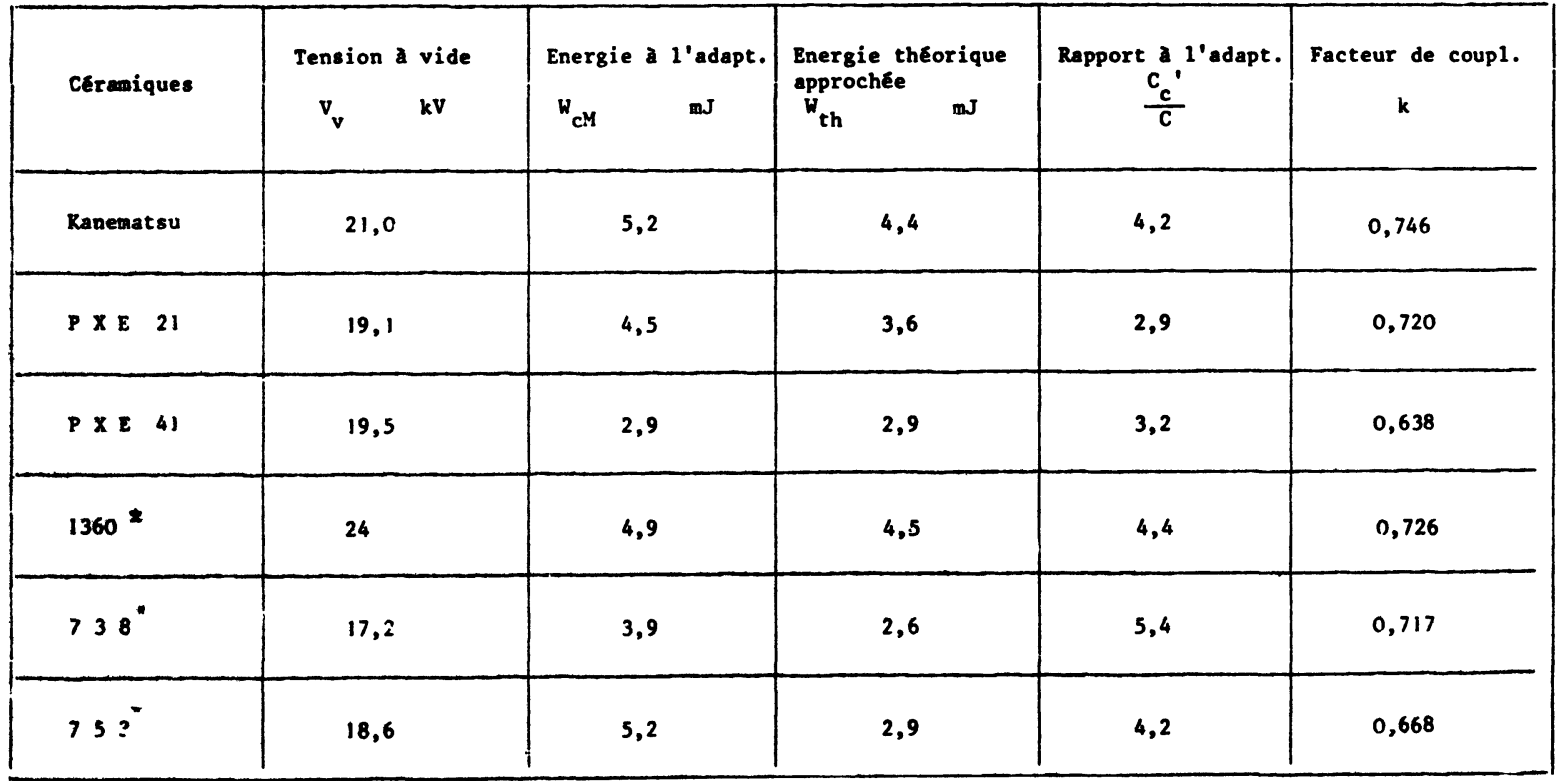

facteur $F$ s'exprime par $F=k^{2} s^{E}$ et varie bien dans le sens de $k$.

3.3 ENERGIES RÉCUPÉRÉES. - Les formules théoriques font apparaitre le terme

$$
W_{0}=\frac{1}{2} F T_{\mathrm{M}}^{2} \cdot v^{\prime}
$$

dans la détermination de l'énergie électrique stockée, avec un coefficient multiplicatif 0,422 ou 1,91 .

On peut déterminer une valeur approchée de la contrainte maximale $T_{\mathrm{M}}$ en utilisant soit la valeur du champ maximum à vide soit celle de la charge maximum en court-circuit. Pour un circuit de charge tel que celui de la figure 3 ou de la figure 5 , la valeur exacte de $T_{\mathrm{M}}$ est comprise entre ces deux déterminations.

Prenons par exemple la détermination par le champ à vide

$$
T_{\mathrm{M}}=\frac{E_{\mathrm{M}}}{g_{33}}=\frac{V_{\mathrm{M}}}{\lg _{33}}
$$


d'où

$$
\frac{\mathrm{W}_{0}}{v^{\prime}} \# \frac{1}{2} g_{33}^{2} \varepsilon^{T} \frac{\mathrm{V}_{\mathrm{M}}^{2}}{1^{2} g_{33}^{2}}
$$

avec

$$
\varepsilon^{T}=C \frac{l}{A}, v^{\prime}=A l, W_{0} \# \frac{1}{2} C V_{\mathrm{M}}^{2} .
$$

Appliquons cette relation pour déterminer une énergie théorique maximum.

- Céramique $801 \quad C=1180 \mathrm{pF} \quad V_{\mathrm{M}}=1750 \mathrm{~V}$ à vide :

- sans self $W_{\mathrm{th}}=760 \mu \mathrm{J}$ comparable à l'énergie mesurée $W_{\mathrm{cm}}=540 \mu \mathrm{J}$,

- avec self $W_{\mathrm{th}}=3,4 \mathrm{~mJ}$ cette valeur est très éloignée de la valeur pratique de $1,28 \mathrm{~mJ}$; cet écart est probablement dû à la détermination approchée de $T_{\mathrm{M}}$.

- Céramiques épaisses : le tableau I donne l'énergie théorique calculée en tenant compte de l'effet de la capacité parasite de $12 \mathrm{pF}$. L'approximation est encore valable dans la plupart des cas.

4. Conclusion. - Une bonne concordance entre l'étude théorique du transfert de l'énergie au cours de la compression et les résultats expérimentaux est observée, tant sur la valeur de la capacité d'adaptation que sur celle de l'énergie maximum récupérée.
Nous remarquons que le choix de la capacité n'est pas critique autour de la valeur théorique car l'énergie varie faiblement dans la zone du maximum.

Par ailleurs, les calculs théoriques montrent que les conditions d'adaptation dépendent peu de la forme de l'impulsion de pression (sinusoïde pure ou composition de plusieurs ondes). Pour des céramiques industrielles dont le coefficient de couplage est compris entre 0,5 et 0,8 la variation de compliance de l'échantillon introduit des modifications de la capacité d'adaptation inférieures à $4 \%$ donc négligeables, compte tenu de la remarque précédente. Quant à l'énergie, elle croit évidemment avec le coefficient de couplage du matériau.

Enfin, une nette amélioration de l'énergie récupérée sur la charge est obtenue par insertion d'une self en série avec le générateur. Ce phénomène s'explique en schématisant la céramique piézoélectrique par un générateur de tension $\lg _{33} T$ d'impédance interne capacitive $(C \omega)^{-1}$; la présence de la self d'accord compense cette capacité et conduit à réaliser un générateur de très faible résistance interne permettant de débiter de plus forts courants. Ce gain en énergie dû à la self est en pratique de 2,5 pour des céramiques de faible épaisseur. Un problème se pose pour les céramiques épaisses de très faible capacité : il faudrait alors utiliser des inductances de très fortes valeurs (de 2 à $5 \mathrm{H}$ pour les échantillons industriels) supportant des tensions de l'ordre de $20 \mathrm{kV}$. Cette contrainte rend alors leur utilisation rédhibitoire.

\section{Bibliographie}

[1] Randeraat, J., Setterington, R. E., Piezoelectric ceramics. Philips Pub. Dep. Electron. Compon. and Materials (1980).

[2] Ohanessian, H., Gonnard, P., Fetiveau, Y., Revue Phys. Appl. Tome 9 (1974) pp. 995-999.

[3] JAfFe, B., CoOK, W. R., JAFFe, H., Piezoelectric Ceramics (Acad. Press. New York) 1971.

[4] Proceedings of the IRE Standards on Piezoelectric Crystals (1949).

[5] Eyraud, L., Eyraud, P., Gonnard, P., Troccaz, M., Ferroelectrics Vol. 31 (1981) pp. 113-116.
[6] Eyraud, L., Eyraud, P., Gonnard, P., Troccaz, M., C.R. Hebd. Séan. Acad. Sci. Paris Série B t. 288 (Fév. 1979), pp. 115-118.

[7] Yadav, B., Indian. J. Phys. V 42 (1969) pp. 649-655.

[8] Chaudhuri, B., Proc. Nat. Inst. Sci. India A V. 35 (1968) pp. 709-718.

[9] Shvedova, L. A., Elektrichestvo no 10 (1971) pp. 27-35. 